\title{
Imaging Tumor Response to Radiation Therapy in Liver Hesheng Wang*
}

Department of Radiation Oncology, University of Michigan, Ann Arbor, MI, 48109, USA

Currently, there are increasing interests in imaging tumor response to radiation therapy (RT) of hepatic cancer. Early detection of tumor response may allow a change in the treatment before completion of the prescribed therapy and maximize therapeutic efficacy of the treatment. Most importantly, once early tumor response indicates achievement of final tumor control, withholding treatment will prevent radiationinduced diseases. Response Evaluation Criteria in Solid Tumors (RECIST) which evaluates tumor response based on change in tumor size is a clinically adopted approach to determine solid tumor responses. But it may take several weeks for RECIST criteria to suggest success or failure of a treatment, which may lead to severe radiation toxicity or tumor progression due to inadequate radiation delivery. Advances of medical imaging allow high-sensitivity and high-specificity assessment of tumor biological process and, therefore, may provide surrogates of tumor response before morphological change occurs.

Liver metastases response to Yttrium-90 radioembolization for 42 patients with unresectable liver metastases was assessed by CT and 18F-FDG PET [1]. The study graded FDG uptake of treated lesions into four categories. In comparison with response detection rates of $6 \%$ using RECIST and 24\% using combined RECIST and necrosis criteria, PET detected tumor response to treatment in a rate of $63 \%$. It was 61-160 days later that CT detected response on the lobes that initially showed response only on PET. The results suggested PET captured tumor response much earlier. In a recent study, Yttrium-90 radioembolization of 58 patients with hepatic metastases from breast cancer was imaged by 18F-FDG PET/CT [2]. 18F-FDG PET was acquired before and 3 month after the treatment. Percentage change in maximal 18F-FDG SUV ( $\mathrm{SUV}_{\text {max }}$ ) relative to the baseline was evaluated. SUV ${ }_{\max }$ assessing responsers showed significantly longer survival ( 65 weeks) than nonresponsers with survival of 43 weeks. The study found the change in SUV max $_{\text {max }}$ measured by $18 \mathrm{~F}-\mathrm{FDG}$ PET before and 3 month after treatment was the only independent predictor of survival of the therapy for patients with hepatic metastases of breast cancer. Compared with morphological imaging such as CT, 18F-FDG PET detects early tumor metabolic change in response to treatment; however, PET is limited for depiction of small tumors partially because of its low spatial resolution.

Diffusion-weighted MR imaging (DWI) and derived Apparent diffusion coefficients (ADC) image mobility of water molecules and have shown potential to detect tumor necrosis and apoptosis resulting from cancer treatment. The feasibility of using ADC change as a surrogate for tumor response to RT was demonstrated by an imaging study of 11 patients treated with six-fraction conformal liver RT [3]. Median ADC in treated tumor VOI kept increasing with the time course of before, one week during, two weeks during and one month after RT. Early increases in mean ADC correlated with higher radiation dose and increased likelihood of response. Tumor response to brachytherapy of colorectal liver metastases was assessed by DWI in a study of 30 patients undergoing single-fraction 192Ir-high-dose-rate brachytherapy [4]. DWI of the liver prior to, 2 days after as well as 3 month after therapy was acquired. Compared with baseline ADC, mean ADC of treated tumors decreased by $11.4 \% 2$ days after treatment, but increased by $28.6 \% 3$ months after the therapy. Therefore, on early in 2 days after therapy, DWI was able to detect tumor response by significant decreasing of mean tumor ADC.

These studies present the ability of imaging for assessment of tumor response to RT for hepatic cancer. However, further development and evaluation of the imaging techniques are in great need, for examples, kinetic modeling analysis of PET, determining optimal timing of imaging assessment of tumor response, and evaluation of sensitivity and specificity of the derived imaging parameters for detecting tumor response. Most of current methods assess tumor response by the changes of derived imaging parameters in tumor VOIs. Heterogeneity within a tumor and across individuals may significantly contribute to obtaining effective and reliable surrogates of tumor response to RT in liver.

\section{References}

1. Miller FH, Keppke AL, Reddy D, Huang J, Jin J, et al. (2007) Response of liver metastases after treatment with yttrium-90 microspheres: role of size, necrosis, and PET. AJR Am J Roentgenol 188: 776-783.

2. Haug AR, Tiega Donfack BP, Trumm C, Zech CJ, Michl M, et al. (2012) 18F-FDG $\mathrm{PET} / \mathrm{CT}$ predicts survival after radioembolization of hepatic metastases from breast cancer. J Nucl Med 53: 371-377.

3. Eccles CL, Haider EA, Haider MA, Fung S, Lockwood G, et al. (2009) Change in diffusion weighted MRI during liver cancer radiotherapy: preliminary observations. Acta Oncol 48: 1034-1043.

4. Wybranski C, Zeile M, Löwenthal D, Fischbach F, Pech, et al. (2011) Value of diffusion weighted MR imaging as an early surrogate parameter for evaluation of tumor response to high-dose-rate brachytherapy of colorectal liver metastases. Radiat Oncol 6: 43.
*Corresponding author: Hesheng Wang, Department of Radiation Oncology, University of Michigan, Ann Arbor, MI, 48109, USA, E-mail: hesheng@med.umich.edu

Received June 28, 2012; Accepted June 29, 2012; Published June 30, 2012

Citation: Wang H (2012) Imaging Tumor Response to Radiation Therapy in Liver. J Nucl Med Radiat Ther S6:e002. doi:10.4172/2155-9619.S6-e002

Copyright: (c) 2012 Wang $\mathrm{H}$. This is an open-access article distributed under the terms of the Creative Commons Attribution License, which permits unrestricted use, distribution, and reproduction in any medium, provided the original author and source are credited. 